\title{
A WEIGHTED POLYNOMIAL INEQUALITY
}

\author{
D. S. LUBINSKY
}

\begin{abstract}
In the theory of orthogonal polynomials for weights with noncompact support, much use is made of inequalities relating weighted integrals of polynomials over infinite and finite ranges. Using a short new method of proof, we show such inequalities hold for very general weights in $L_{p}$ and certain Orlicz spaces.
\end{abstract}

Introduction. Let $\mathbf{P}_{n}$ denote the class of real polynomials of degree at most $n$, and let $C_{1}, C_{2}, \ldots$ denote positive constants independent of $n$ and $P \in \mathbf{P}_{n}$. Freud made extensive use of the following inequality [3, Theorem 2.6]:

$$
\|P W\|_{L_{2}(-\infty, \infty)} \leqslant\left(1+C_{1} e^{-C_{2} n}\right)\|P W\|_{L_{2}\left(-C_{3} q_{2 n}, C_{3} q_{2 n}\right)}
$$

$P \in \mathbf{P}_{n}$. Here $W=\exp (-Q)$ where $Q$ and $Q^{\prime}$ satisfy certain restrictions and $q_{n}$ is the positive root of $q_{n} Q^{\prime}\left(q_{n}\right)=n$ and of (4) below. The corresponding $L_{\infty}$ inequality for the Hermite weight $W=\exp \left(-x^{2} / 2\right)$ appears in [2]. Similar inequalities for special weights have frequently been used by Freud and Nevai.

For the Hermite weight, $1 \leqslant p \leqslant \infty$ and $0<r<\infty$, Zalik [6, Theorems 1 and 4] proved

$$
\begin{aligned}
\left\|x^{r} P(x) W(x)\right\|_{L_{p}(-\infty, \infty)} & \leqslant C_{4} n^{r / 2}\|P(x) W(x)\|_{L_{p}(-\infty, \infty)}, \\
\|P W\|_{L_{p}(-\infty, \infty)} & \leqslant C_{5}\|P W\|_{L_{p}(-4 \sqrt{n}, 4 \sqrt{n}),}
\end{aligned}
$$

$P \in \mathbf{P}_{n}$ with $C_{5}$ independent of $p \in[1, \infty]$. In [4, Theorem 5.1], (1) and (2) were generalized for $p=2$ :

$$
\left.\|g P W\|_{L_{2}(-\infty, \infty)} \leqslant\left(1+C_{6}(0.9)^{2 n}\right)\left|3 q_{2 n}\right|^{\gamma}\|P W\|_{L_{2}\left(-3\left|q_{2 n}\right|, 3\left|q_{2 n}\right|\right.}\right),
$$

$P \in \mathbf{P}_{n}, n \geqslant n_{1}$. The weight $W$ in [4] satisfied the following conditions:

$W$ is nonnegative in $\mathbf{R}$ and measurable, with all moments finite, and for some $A, B>0, W(x)$ is continuous for $|x| \geqslant A$ and

$$
\min _{A \leqslant|u| \leqslant|x|} W(u) \geqslant B W(x)>0, \quad|x| \geqslant A .
$$

In particular, the last inequality holds if $W(x)$ is even and nonincreasing for large $|x|$.

Received by the editors October 11, 1983.

1980 Mathematics Subject Classification. Primary 41A17; Secondary 26D05, 42C99.

Key words and phrases. Weighted polynomial inequality, noncompactly supported weight.

(C1984 American Mathematical Society $0002-9939 / 84 \$ 1.00+\$ .25$ per page 
The numbers $q_{t}, t>0$, are defined as the root of smallest absolute value of the equation

$$
W\left(q_{t}\right)\left|q_{t}\right|^{t}=\max _{|x| \geqslant A}\left\{W(x)|x|^{t}\right\},
$$

taken as positive if both negative and positive values may be chosen. Finally, in [4] the function $g$ satisfies the following conditions with $D=1$ :

$$
\begin{gathered}
g: \mathbf{R} \rightarrow \mathbf{R} \text { is measurable and for some } D>0 \text { and } \gamma \geqslant 0, \\
|g(x)| \leqslant D(\max \{1,|x|\})^{\gamma}, \quad x \in \mathbf{R} .
\end{gathered}
$$

The proofs of these inequalities in $[\mathbf{2 , 3}, \mathbf{4 , 6}$ depend upon estimates for the Christoffel functions, properties of the Hermite weight and convexity arguments. In this note we use Cartan's Lemma on small values of polynomials [1, p. 174] to prove inequalities such as

Theorem A. Let $W$ and $g$ be as in (3) and (5) respectively. Let $0<\theta \leqslant 1$. There exist $\rho \in(0,1)$ and $n_{1}$ depending on $W, g$ and $\theta$ but not on $n, p$ or $P$ such that

$$
\begin{aligned}
\|g P W\|_{L_{p}(-\infty, \infty)} \leqslant\left(1+\rho^{n}\right)^{1 / \theta} D\left(11\left|q_{2 n}\right|\right)^{\gamma}\|P W\|_{L_{p}\left(-11\left|q_{2 n}\right|, 11\left|q_{2 n}\right|\right)}, & \\
\theta & \leqslant p \leqslant \infty, P \in \mathbf{P}_{n}, n \geqslant n_{1} .
\end{aligned}
$$

For the Hermite weight, Theorem A removes the restriction $p \geqslant 1$ in (1) and (2) though $11 q_{2 n}=11 \sqrt{2 n}>4 \sqrt{n}$.

Inequalities such as the one in Theorem $A$ are useful because they reduce problems over an infinite interval to problems on a finite interval, thereby enabling one to apply results for orthogonal polynomials on a finite interval, such as Szego's theory, to weights over an infinite interval. Further, they are essential in estimating the Christoffel functions and in investigating mean convergence of Lagrange interpolation and orthonormal expansions for weights on the whole real line.

Recently, Mhaskar and Saff [5] undertook a penetrating investigation of the $L_{\infty}$ version of the inequalities in Theorem $\mathrm{A}$ for $g=1$ and the weights $W_{\alpha}(x)=$ $\exp \left(-|x|^{\alpha}\right), \alpha>0$, using complex methods. They showed [5, Theorem 2.7] that for $P \in \mathbf{P}_{n}$

$$
\left\|P W_{\alpha}\right\|_{L_{\infty}(-\infty, \infty)}=\left\|P W_{\alpha}\right\|_{L_{\infty}\left(-a_{n}(\alpha), a_{n}(\alpha)\right)}
$$

where $a_{n}(\alpha)=\left(n / \lambda_{\alpha}\right)^{1 / \alpha}$ cannot be reduced. Further they obtained an $L_{p}$ inequality [5, Lemma 6.3] like that in Theorem A, for $g=1$, with constants depending on $p$ $\in(0, \infty]$ and implicit assumptions on the weight $W[5,(6.13-6.15)]$. It is unlikely that (6.13) in [5] will hold whenever (3) holds and (6.15) in [5] is not satisfied by weights like

$$
W(x)=\exp (-|\log | x|||\log | \log |x|||)
$$

which satisfy (3) above. However, it seem.s certain that there are weights not satisfying (3) but for which (6.13-6.15) in [5] are true. 
2. The general inequality. Throughout, let $\psi: \mathbf{R} \rightarrow[0, \infty)$ be an even function, monotone increasing on $(0, \infty)$, continuous in $\mathbf{R}$, with $\psi(0)=0$. Let

$$
\psi^{*}(y)=\sup _{x>0} \psi(x y) / \psi(x), \quad y \in \mathbf{R},
$$

and assume this is finite in $\mathbf{R}$. It is trivially finite in $[0,1]$. We also assume there exists $\eta>0$ such that for $r \in[0,1]$,

$$
I[r]=\int_{0}^{r} \psi^{*}(v) v^{-1-\eta} d v<\infty
$$

THEOREM B. Let $W$ and $g$ be as in (3) and (5) respectively. Let $\psi$ be as above and $\psi^{*}$ be as in (7) and (8). Let $0<\varepsilon \leqslant 14$. There exist $C_{7}$ and $n_{1}$ depending on $g, W, A, B, \varepsilon, D, \gamma$ but independent of $\psi$ such that for $n \geqslant \max \left\{n_{1},\left(\eta^{-1}+\gamma\right) / \varepsilon\right\}$ and all $P \in \mathbf{P}_{n}$,

(9) $\int_{-\infty}^{\infty} \psi(g P W) d x \leqslant \psi^{*}\left(D \xi_{n}^{\gamma}\right) \int_{-\xi_{n}}^{\xi_{n}} \psi(P W) d x\left\{1+C_{7} n^{-1} I\left[B^{-1}(0.99)^{n}\right]\right\}$

where

$$
\xi_{n}=11^{1 / \varepsilon}\left|q_{n(1+\varepsilon)}\right|, \quad n \geqslant n_{1} .
$$

Proof. Write $H=11^{1 / \varepsilon}$ and $\chi=\left|q_{n(1+\varepsilon)}\right|$. Let $P \in \mathbf{P}_{n}, P \not \equiv 0$, and write

$$
P(u)=c \prod_{i=1}^{m}\left(u-x_{i}\right)
$$

where $m \leqslant n,\left|x_{i}\right| \leqslant H \chi$ for $1 \leqslant i \leqslant j$ and $\left|x_{i}\right|>H \chi$ for $j<i \leqslant m$. Then if $|x| \geqslant H \chi$, $|u| \leqslant \chi$ and $j<i \leqslant m$, we have

$$
\frac{\left|x-x_{i}\right|}{\left|u-x_{i}\right|} \leqslant \frac{1+|x| /(H \chi)}{1-|u| /(H \chi)} \leqslant \frac{2|x|}{(H-1) \chi} .
$$

Let $r=(1-\delta) /(2 e)$ where $\delta>0$ is chosen so small that $2 /(11 r)<0.99$. We see $H-1>r$ for $0<\varepsilon \leqslant 14$. Hence,

$$
\frac{|P(x)|}{|P(u)|} \leqslant\left(\frac{2|x|}{r \chi}\right)^{m-j}(2|x|)^{j} /\left|\prod_{i=1}^{j}\left(u-x_{i}\right)\right| \leqslant\left(\frac{2|x|}{r \chi}\right)^{m},
$$

for $|x| \geqslant H \chi,|u| \leqslant \chi$ and $u \notin \mathscr{S}$, where $\mathscr{S}$ is open and has linear measure at most 4 er $\chi$. Here we have used Cartan's Lemma on small values of polynomials (see, for example, [1, p. 174]). Let $\mathscr{M}=[-\chi, \chi] \backslash(\mathscr{S} \cup(-A, A))$. Choose $\sigma= \pm 1$ so that $\sigma \chi=q_{n(1+\varepsilon)}$. By (5) and (11), and as $m \leqslant n$, we have for $|x| \geqslant H \chi>1, u \in \mathscr{M}$,

$$
\begin{aligned}
|g(x) P(x) W(x)| & \leqslant \frac{D}{B}(H \chi)^{\gamma}\left(\frac{|x|}{H \chi}\right)^{\gamma-n \varepsilon}\left(\frac{2}{11 r}\right)^{n}|P(u)| B W(\sigma \chi) \frac{|x|^{n(1+\varepsilon)} W(x)}{\chi^{n(1+\varepsilon)} W(\sigma \chi)} \\
& \leqslant \frac{D}{B} \xi_{n}^{\gamma}\left(\frac{|x|}{\xi_{n}}\right)^{\gamma-n \varepsilon}(0.99)^{n}|P(u)| W(u),
\end{aligned}
$$


by (3), (4) and (10). Using (7) twice, we see

$$
\begin{array}{r}
\psi(g(x) P(x) W(x)) \\
\leqslant \psi^{*}\left(D \xi_{n}^{\gamma}\right) \psi^{*}\left(B^{-1}\left(|x| / \xi_{n}\right)^{\gamma-n \varepsilon}(0.99)^{n}\right) \psi(P(u) W(u)), \\
|x| \geqslant \xi_{n}, u \in \mathscr{M} .
\end{array}
$$

Further, as $\mathscr{M}$ has linear measure at least $2 \chi-4 \operatorname{er} \chi-2 A=2(\delta \chi-A)$, we have for $\chi>A / \delta$ and a certain $u \in \mathscr{M}$,

$$
\psi(P(u) W(u)) \leqslant \frac{1}{2(\delta \chi-A)} \int_{\mathscr{M}} \psi(P(v) W(v)) d v .
$$

Then (12) and (13) yield

$$
\begin{aligned}
\int_{|x| \geqslant \xi_{n}} \psi & (g P W) d x /\left\{\psi^{*}\left(D \xi_{n}^{\gamma}\right) \int_{-\xi_{n}}^{\xi} \psi(P W) d x\right\} \\
& \leqslant \frac{1}{(\delta \chi-A)} \int_{\xi_{n}}^{\infty} \psi^{*}\left(B^{-1}\left(\frac{x}{\xi_{n}}\right)^{\gamma-n \varepsilon}(0.99)^{n}\right) d x \\
& =\frac{\xi_{n}}{\delta H^{-1} \xi_{n}-A} \frac{\left[B^{-1}(0.99)^{n}\right]^{1 /(n \varepsilon-\gamma)}}{n \varepsilon-\gamma} \int_{0}^{B^{-1}(0.99)^{n}} \psi^{*}(v) v^{-1-1 /(n \varepsilon-\gamma)} d v \\
& \leqslant C_{7} n^{-1} I\left[B^{-1}(0.99)^{n}\right]
\end{aligned}
$$

for $n \geqslant n_{1}$ such that also $(n \varepsilon-\gamma)^{-1}<\eta$. Here $n_{1}$ and $C_{7}$ have the properties in the statement of Theorem B. The result follows by a single application of (5) and (7).

Proof of Theorem A. Let $\eta=\theta / 2$. Let $\psi(t)=|t|^{p}$ where $\infty>p \geqslant \theta$. We see then $\psi^{*}(t)=|t|^{p}$ and $\psi^{*}(t) \leqslant t^{\theta}, t \in[0,1]$, so that, for $n \geqslant n_{2}=n_{2}(B)$,

$$
I\left[B^{-1}(0.99)^{n}\right] \leqslant(2 / \theta)\left(B^{-1}(0.99)^{n}\right)^{\theta / 2},
$$

the right side being independent of $p \geqslant \theta$. Substituting in (9) and (10) with $\varepsilon=1$ and taking $p$ th roots, we obtain (6) for $\theta \leqslant p<\infty$. As (5) holds, and as the constants in (6) are independent of $\psi(t)=|t|^{p}$, we can let $p \rightarrow \infty$ to obtain the inequality for $p=\infty$ also.

Remarks. (a) We note that $\psi(t)=|t|^{p}(1+|\log | t||)^{q}, p>0, q \in \mathbf{R}$, also satisfies (8), with $\psi^{*}(t)=\psi(t)$ if $q \geqslant 0$ and $\psi^{*}(t)=|t|^{p}$ if $q<0$. However, $\psi$ is monotone increasing throughout $(0, \infty)$ only if $p \geqslant|q|$.

(b) One cannot in general replace $\xi_{n}$ in (9) and (10) by $\delta\left|q_{n}\right|$ with $\delta$ arbitrarily small [4, Lemma 5.2].

(c) Theorem B is useful in finding upper bounds for generalized Christoffel numbers

$$
\lambda_{n}(W ; \psi ; \Phi)=\inf _{P \in \mathbf{P}_{n-1}} \int_{-\infty}^{\infty} \psi(P W) d x / \psi(\Phi(P))
$$

where $\Phi$ is a real functional defined on all polynomials. 
(d) If one weakens (3) to allow

$$
\min _{A \leqslant|u| \leqslant|x|} W(u) \geqslant h(x) W(x), \quad|x| \geqslant A,
$$

where $h$ is even, positive and nonincreasing, and $\lim _{\sup _{n \rightarrow \infty}} h\left(\xi_{n}\right)^{1 / n}<\infty$, then (9) is still valid if one replaces $B^{-1}$ by $h\left(\xi_{n}\right)^{-1}$. The continuity of $W(x)$ for $|x| \geqslant A$ may also be dropped, provided (3) and the definition (4) of $q_{t}$ are suitably modified.

\section{REFERENCES}

1. G. A. Baker, Jr., Essentials of Padé approximants, Academic Press, New York, 1975.

2. G. Freud, On two polynomial inequalities. I, Acta Math. Acad. Sci. Hungar. 22 (1971), 109-116.

3. __ On polynomial approximation with respect to general weights, Lecture Notes in Math., (H. G. Garnir, K. R. Unni, J. H. Williamson, eds.), vol. 399, Springer, Berlin, 1974, pp. 149-179.

4. D. S. Lubinsky, Estimates of Freud-Christoffel functions for some weights with the whole real line as support, J. Approx. Theory (to appear).

5. H. N. Mhaskar and E. B. Saff, Extremal problems for polynomials with exponential weights, Trans. Amer. Math. Soc (to appear).

6. R. Zalik, Inequalities for weighted polynomials, J. Approx. Theory 37 (1983), 137-146.

National Research InStitute for Mathematical Sciences of the CSIR, P. O. Box 395, Pretoria 0001, RePublic OF SOUTh Africa 